\title{
AN ALGORITHM FOR FAULT NODE RECOVERY OF WIRELESS SENSOR NETWORK
}

\author{
Ashwini Yenegur ${ }^{1}$, Basawaraj.S.Mathpati ${ }^{2}$ \\ ${ }^{I}$ PG student, CSE Department, Appa Institute of Engineering and Technology, Gulbarga, Karnataka, India \\ ${ }^{2}$ PG Head, CSE Department, Appa Institute of Engineering and Technology, Gulbarga Karnataka, India
}

\begin{abstract}
Sensors in a wireless sensor networks (WSNs) are having tendency to fail, due to the energy depletion, hardware failures, environmental conditions etc. Fault tolerance is one of the critical issues in WSNs. The existing fault tolerance mechanisms either consume significant extra energy to detect and recover from the failures or need to use additional hardware and software resources. The proposed algorithm enhances the lifetime of a sensor nodes shut down and it depends on ladder diffusion algorithm combined with the genetic algorithm. It can result in fewer replacements of sensor nodes with more reused routing paths and also increases the number of active nodes, reduce the rate of data loss with reduced energy consumption.
\end{abstract}

Keywords: Wireless sensor networks, genetic algorithm, ladder diffusion algorithm

\section{INTRODUCTION}

Wireless sensor networks (WSNs) are composed of massive, small and low-cost sensor nodes deployed in a monitoring region, forming a multi-hop self-organized network system through wireless communication. The targets is to cooperatively sense, collect and process the information about objects in the coverage area, and then send it to the observer for processing and analyzing. It is a system with multifunctional and low energy consumption.

In sensor networks, each sensor node has limited wireless computational power to process and transfer the live data to the base station or data collection center [2]. Therefore, to increase the sensor area and the transmission area [1], the wireless sensor network usually contains many sensor nodes. Generally, each sensor node has a low level of battery power that cannot be replenished. When the energy of a sensor node is exhausted, wireless sensor network leaks will appear, and the failed nodes will not relay data to the other nodes during transmission processing. Thus, the other sensor nodes will be burdened with increased transmission processing. This proposed algorithm enhances the lifetime of a wireless sensor network (WSN) when some of the sensor nodes shut down, either because they no longer have battery energy or they have reached their operational threshold. Using the proposed algorithm can result in fewer replacements of sensor nodes and more reused routing thus, the algorithm not only enhances the WSN lifetime but also reduces the cost of replacing the sensor nodes.

\section{RELATED WORK}

Many techniques have been proposed till now for fault detection and recovery. Sony Jia et al. [1] proposed a Recovery Algorithm based on Minimum Distance Redundant Nodes (MDRN). By employing redundant nodes carefully, the recovery algorithm is deployed on the sink node with unconstrained energy consumption which knows the locations of all active nodes and redundant nodes in the WSNs. Simulation results demonstrate that, by choosing appropriate number of redundant nodes, this algorithm will have great recovery accuracy and coverage quality, also achieve the purpose of prolonging the lifecycle of WSNs.

Muhammed Asim et al. [2] extended the cellular approach and proposed a new fault management mechanism to deal with fault detection and recovery of wsn. They proposed a hierarchical structure to properly distribute fault management tasks among sensor nodes by introducing more 'selfmanaging' functions. The proposed failure detection and recovery algorithm has been compared with some existing related work and proven to be more energy efficient. Sanam Hasseini et al. [3] explained that a wireless sensor network composed of many sensor nodes which are used to monitor unavailable and harsh environments. Because these nodes are too small and battery operated which have limited energy, faults may occur. Fault tolerance is one of the most important issues in wireless sensor networks and must be increased as much as possible to avoid faults. In wireless sensor networks which use clustering architecture, the role of cluster head is very important and critical and fault tolerance in cluster head must be increased. Different approaches for increasing fault tolerance and fault management presented that have advantages and disadvantages. An approach for fault 
management in cluster head is to recover members of faulty cluster with specifying new cluster head for them. In this paper a new recovery algorithm based on inheritor selection is proposed. Previous algorithms do cluster head selection when each fault occurs but the proposed algorithm does this selection once and can select cluster head rapidly and without too much calculation. Simulations results show that the proposed algorithm has better performance in contrast to previous algorithms.

Charu virmani and Khushboo Garg [4] discussed about already implemented algorithms and existing approaches of network fault management and compare their features for an effective one. Prasenjitchanak et al. [5] proposed an energy efficient node fault diagnosis and recovery for wireless sensor networks referred as fault tolerant multipath routing scheme for energy efficient wireless sensor network (FTMRS).The FTMRS is based on multipath data routing scheme. One shortest path is use for main data routing in FTMRS technique and other two backup paths are used as alternative path for faulty network and to handle the overloaded traffic on main channel. Shortest path data routing ensures energy efficient data routing. The performance analysis of FTMRS shows better results compared to other popular fault tolerant techniques in wireless sensor networks.

Jian yang et al. [6] proposed a strategy for the WSN in that situation. Based on the Support Vector Machine (SVM), we suggested a decision-function. In this function, by using support vector we can balance multiple causes of a fault except energy. This light calculation can make fault management of WSN more efficient, practical and easydeployed to improve the lifetime and robustness of the whole network. The simulation experiment showed that, based on the $\mathrm{v}$-SVC, the $\mathrm{CH}$ replacing and re-clustering algorithm are more efficient than the traditional solutions in way of optimizing the training sets and kernel function. Beenu Baby and Joe Mathew Jacob [8] proposed a fault tolerant sleep scheduling technique, which schedules the sleep and wake up in the network and also propose a fault tolerance in the corrupted nodes in the network. For the alarm transmission, propose two traffic paths called up link and down link. Schedule a specific wake up patterns for the sleep schedule technique. Fault tolerance mechanism adopts fault detection and fault recovery of the sensor nodes.

J. A. Carballido et al. [9] discussed the foundations and implementation of a genetic algorithm (GA) for instrumentation purposes. The GA constitutes an initialization module of a decision support system for sensor network design. The method development entailed the definition of the individual's representation as well as the design of a graphbased fitness function, along with the formulation of several other ad hoc implemented features. The performance and effectiveness of the GA were assessed by initializing the instrumentation design of an ammonia synthesis plant. The initialization provided by the GA succeeded in accelerating the sensor network design procedures. It also accomplished a great improvement in the overall quality of the resulting instrument configuration. Therefore, the GA constitutes a valuable tool for the treatment of real industrial problems.

J. H. Ho et al. [13] proposed an algorithm based on ladder diffusion and ACO and is proposed to solve the power consumption and transmission routing problems in wireless sensor networks. The proposed ladder diffusion algorithm is employed to route paths for data relay and transmission in wireless sensor networks, reducing both power consumption and processing time to build the routing table and simultaneously avoiding the generation of circle routes. Moreover, to ensure the safety and reliability of data transmission, our algorithm provides backup routes to avoid wasted power and processing time when rebuilding the routing table in case part of sensor nodes are missing. J. H. Ho et al. [14] proposed GD algorithm which creates the routing for each sensor node but also identifies a set of neighbor nodes to reduce the transmission loading. Each sensor node can select a sensor node from the set of neighbor nodes when its grade table lacks a node able to perform the relay. The GD algorithm can also record some information regarding the data relay. Then, a sensor node can select a node with a lighter loading or more available energy than the other nodes to perform the extra relay operation. That is, the GD algorithm updates the routing path in real time, and the event data is thus sent to the sink node quickly and correctly.

C. Intanagonwiwat et al. [15] explored the directed-diffusion paradigm for such coordination. Directed diffusion is datacentric in that all communication is for named data. All nodes in a directed-diffusion-based network are application aware. This enables diffusion to achieve energy savings by selecting empirically good paths and by caching and processing data innetwork (e.g., data aggregation). They explore and evaluated the use of directed diffusion for a simple remote-surveillance sensor network analytically and experimentally. This evaluation indicates that directed diffusion can achieve significant energy savings and can outperform idealized traditional schemes (e.g., omniscient multicast) under the investigated scenarios.

\section{PROPOSED SYSTEM}

This paper proposes an algorithm for WSNs based on the ladder diffusion algorithm combined with the genetic algorithm. The flow chart is shown in Fig. 1.Here the ladder diffusion algorithm is employed to route paths for data relay and transmission in wireless sensor networks, reducing both power consumption and processing time to build the routing table and simultaneously avoiding the generation of circle routes. Moreover, to ensure the safety and reliability of data transmission, ladder diffusion algorithm provides backup routes to avoid wasted power and processing time when 
rebuilding the routing table in case part of sensor nodes are missing. In the proposed algorithm, the number of nonfunctioning sensor nodes is calculated during the wireless sensor network operation, and the parameter Bth is calculated according to (1).

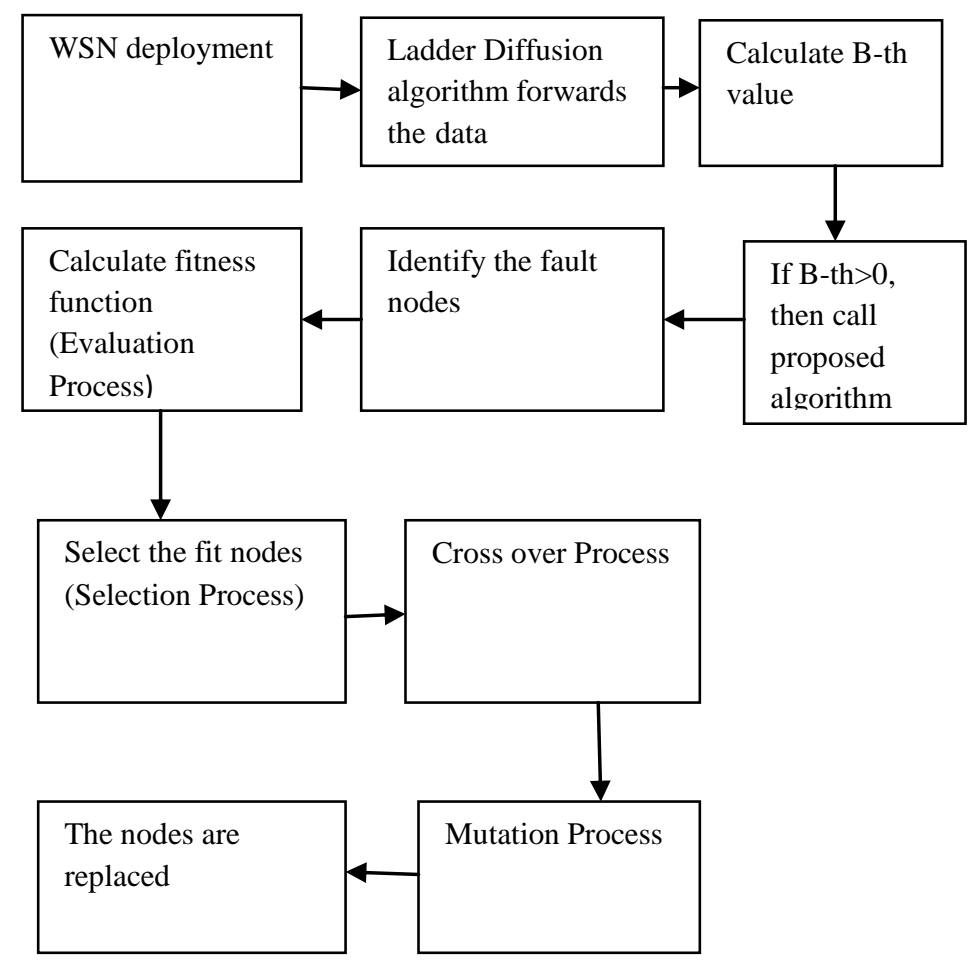

Fig 1 Block Diagram of the proposed algorithm

In Fig. 1, the algorithm creates the grade value, routing table, a set of neighbor nodes, and payload value for each sensor node, using the ladder diffusion algorithm. The sensor nodes transfer the event data to the sink node according to the LD algorithm when events appear. Then, Bth is calculated according to (1) in the proposed algorithm. If Bth is larger than zero, the algorithm will be invoked and replace nonfunctioning sensor nodes by functional nodes selected by the genetic algorithm. Then the wireless sensor network can continue to work as long as the operators are willing to replace sensors

$$
\begin{aligned}
& \mathrm{Bth}=\sum_{\mathrm{i}=1}^{\max \left(g^{\prime} \operatorname{lid}^{2}\right)} T i
\end{aligned}
$$

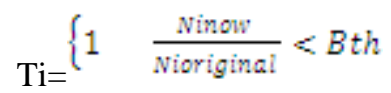

$$
\begin{aligned}
& 0 \text { otherwise }
\end{aligned}
$$

In (1), Grade is the sensor node's grade value. The variable Nioriginal is the number of sensor nodes with the grade value i . The variable Ninow is the number of sensor nodes still functioning at the current time with grade value $i$. The parameter $\beta$ is set by the user and must have a value between 0 and 1 . If the number of sensor nodes that function for each grade is less than $\beta$, Ti will become 1 , and Bth will be larger than zero. Then, the algorithm will calculate the sensor nodes to replace using the genetic algorithm. The parameters are encoded in binary string and serve as the chromosomes for the GA. The elements (or bits), i.e., the genes, in the binary strings are adjusted to minimize or maximize the fitness value. The fitness function generates its fitness value, which is composed of multiple variables to be optimized by the GA. At each iteration of the GA, a predetermined number of individuals will produce fitness values associated with the chromosomes.

There are 5 steps in the genetic algorithm: Initialization, Evaluation, Selection, Crossover, and Mutation. Descriptions of the steps follow.

\subsection{Initialization}

In the initialization step, the genetic algorithm generates chromosomes, and each chromosome is an expected solution. The number of chromosomes is determined according to the population size, which is defined by the user. Each chromosome is a combination solution, and the chromosome length is the number of sensor nodes that are depleted or nonfunctioning. The elements in the genes are either 0 or 1.A 1 means the node should be replaced, and a 0 means that the node will not be replaced

\subsection{Evaluation}

In general, the fitness value is calculated according to a fitness function, and the parameters of the fitness function are the chromosome's genes. However, we cannot put genes directly into the fitness function in the proposed algorithm, because the genes of the chromosome are simply whether the node should be replaced or not and the goal is also to reuse the most routing paths and to replace the fewest sensor nodes. Hence, the number of routing paths available if some nonfunctioning sensor nodes are replaced is calculated, and the fitness function is shown as (3)

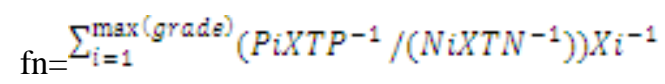

In (3):

$\mathrm{Ni}=$ the number of replaced sensor nodes and their grade value at $\mathrm{i}$.

$\mathrm{Pi}=$ the number of re-usable routing paths from sensor nodes with their grade value at $i$.

$\mathrm{T} \mathrm{N}=$ total number of sensor nodes in the original WSN. $\mathrm{T} \mathrm{P}=$ total number of routing paths in the original WSN.

In (3), a high fitness value is sought because the WSN is looking for the most available routing paths and the least number of replaced sensor nodes. 


\subsection{Selection}

The selection step will eliminate the chromosomes with the lowest fitness values and retain the rest. We use the elitism strategy and keep the half of the chromosomes with better fitness values and put them in the mating pool. The worse chromosomes will be deleted, and new chromosomes will be made to replace them after the crossover step.

\subsection{Crossover}

The crossover step is used in the genetic algorithm to change the individual chromosome. In this algorithm, we use the onepoint crossover strategy to create new chromosomes. Two individual chromosomes are chosen from the mating pool to produce two new offspring. A crossover point is selected between the first and last genes of the parent individuals. Then, the fraction of each individual on either side of the crossover point is exchanged and concatenated. The rate of choice is made according to roulette-wheel selection and the fitness values.

\subsection{Mutation}

The mutation step can introduce traits not found in the original individuals and prevents the GA from converging too fast. In this algorithm, we simply flip a gene randomly in the chromosome. The chromosome with the best fitness value is the solution after the iteration. The proposed algorithm will replace the sensor nodes in the chromosome with genes of 1 to extend the WSN lifetime.

\section{SIMULATION}

Simulation of the proposed algorithm will be performed with the help of NS-2 and the simulation results will show how the faulty sensor nodes are recovered by using most reused paths and these results are compared with existing models.

\section{CONCLUSIONS}

In real wireless sensor networks, the sensor nodes use battery power supplies and thus have limited energy resources. In addition to the routing, it is important to research the optimization of sensor node replacement, reducing the replacement cost, and reusing the most routing paths when some sensor nodes are nonfunctional. This paper proposes an algorithm for WSN based on the ladder diffusion algorithm combined with a genetic algorithm. The proposed algorithm requires replacing fewer sensor nodes and reuses the most routing paths, increasing the WSN lifetime and reducing the replacement cost.

\section{REFERENCES}

[1] Sony Jia, Wang Bailing, Ping Xiyar, Li Jianfeng and Zhong cheng, "A Recovery Based on Minimum Distance redundant Nodes Fault Management in WSNs," International Journal of control and automation, vol 6,No.2 April 2013.

[2] Muhammed Asim, Hala Mokhtar and Madjid Merabti, "A self-managing fault management mechanism for wireless sensor networks," International Journal of Wirele\& Mobile Networks (IJWMN) Vol.2, No.4, November 2010.

[3] Sanam Hasseini, Elmira Moghaddami Khalilzad, "MISR: Multiple Inheritor Selection for Recovery of Nodes in Faulty Cluster in Wireless Sensor Networks," IJCSI International Journal of Computer Science Issues, Vol. 9, Issue 4, No 1, July 2012.

[4] Charu virmani, Khushboo Garg, "Comparative study of fault management algorithms in WSNs," IJERT VOL.1 may 2012.

[5] Prasenjitchanak, Tuhinasamanta, Indrajit Banerjee, "Fault tolerant multipath routing scheme for energy efficient WSNs", 'IJWMN vol 5.No.2 April 2013.

[6] Jian yang, Zhenzhang ye, Zhiyou Ouyang, "A stratergy for fault recovery of wireless sensor networks based on v-svc," Journal of Information and computational science April 2013.

[7] Lilia Paradis and Qi Han, "A survey of fault management in WSNs," Journal of Network and systems management, 2007.

[8] Beenu Baby and Joe Mathew Jacob, "A self-managing fault management in WSNs," ISSN vol 1 July 2013.

[9] Carballido, I. Ponzoni, and N. B. Brignole, "CGD-GA: A graph based genetic algorithm for sensor network design," Inf. Sci., vol. 177, no. 22, pp. 5091-5102, 2007.

[10] F. C. Chang and H. C. Huang, "A refactoring method for cache-efficient swarm intelligence algorithms," Inf. Sci., vol. 192, no. 1, pp. 39-49, Jun. 2012.

[11] S. Corson and J. Macker, "Mobile Ad Hoc Networking (MANET): Routing Protocol Performance Issues and Evaluation Considerations". New York, NY, USA: ACM, 1999.

[12] Z. He, B. S. Lee, and X. S. Wang, "Aggregation in sensor networks with a user-provided quality of service goal," Inf. Sci., vol. 178, no. 9, pp. 2128-2149, 2008.

[13] J. H. Ho, H. C. Shih, B. Y. Liao, and S. C. Chu, "A ladder diffusion algorithm using ant colony optimization for wireless sensor networks," Inf. Sci., vol. 192, pp. 204-212, Jun. 2012.

[14] C. Intanagonwiwat, R. Govindan, D. Estrin, J. Heidemann, and F. Silva, "Directed diffusion for wireless sensor networking," IEEE/ACM Trans.Netw., vol. 11, no. 1, pp. 2-16, Feb. 2003. 